\title{
CLINICOPATHOLOGICAL SIGNIFICANCE OF BRAF (V600E), NRAS (Q61K) AND TERT (C228T, C250T AND SNP RS2853669) MUTATIONS IN BULGARIAN PAPILLARY THYROID CARCINOMA PATIENTS
}

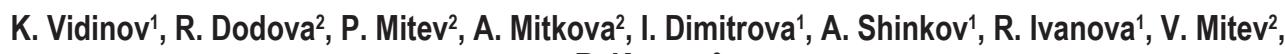 \\ R. Kaneva ${ }^{2}$ \\ 1Department of Endocrinology, Medical Faculty, Medical University - Sofia, Bulgaria \\ ${ }^{2}$ Molecular Medicine Center, Department of Medical Chemistry and Biochemistry, \\ Medical University - Sofia, Bulgaria
}

\begin{abstract}
Introduction: Thyroid carcinoma is the most common endocrine cancer. Some somatic mutations in genes (BRAF, NRAS and TERT) involved in key signaling pathways and genome stability have been recently identified to play an important role in its development. Very little research has been done on their frequency and clinical relevance in Bulgarian patients with papillary thyroid cancer (PTC). This study is focused on investigating somatic mutation frequency in Bulgarian patients with PTC and their association with clinicopathologic features. Material and Methods: The study included 50 PTC from Bulgarian patients analyzed for mutations in BRAF (V600E), NRAS (Q61K), single nucleotide polymorphism (SNP) rs2853669 and TERT (C228T and C250T) genes by Sanger sequencing. The results were interpreted using Benchling and SeqScape software, and statistical analysis performed with SPSS. Results: In the studied PTC group BRAF(V600E) and TERT (C228T) mutations were found with frequency of $24 \%$ and $2 \%$, respectively. Co-occurrence of both mutations was found in 1 patient (2\%). The mutations Q61K (NRAS), and C250T (TERT) were not detected. The SNP rs2853669 was found in 18 patients (52.9\%). Correlation analysis with the clinical characteristics of the patients revealed statistically significant association with larger size of the tumor for $B R A F(V 600 E)$ and smaller tumor size for rs2853669. Conclusion: In the present pilot study, we found that BRAF(V600E) and rs2853669 in TERT are common among PCT patients. While the presence of BRAF V600E mutation was associated with large tumors, the presence of rs2853669 in TERT was found in the majority of PCT below $2 \mathrm{~cm}$. More extensive molecular genetic analysis of TERT, BRAF or RAS mutations in larger sample is needed to further elucidate the clinically important diagnostic and prognostic biomarkers for thyroid cancer.
\end{abstract}

Key words: papillary thyroid cancer, BRAF, NRAS, SNP rs2853669, TERT

Corresponding author: Kalin Vidinov, MD, PhD, Department of Endocrinology, Medical University - Sofia, USBALE "Acad. Iv. Penchev" Hospital - Sofia, Zdrave 2 St, 1463 Sofia, Bulgaria, Tel: +3598956063, E-mail: kalin_vi@hotmail.com ORCID: https://orcid.org/0000-0002-4621-8895

RECEIVED: 14 January 2021, ACCEPTED: 20 January 2021 


\section{INTRODUCTION}

$\mathrm{O}$ ver the last decade, the understanding of thyroid oncogenesis has developed rapidly. This is due to significant technological advances and the widespread use of high-performance molecular biological assays for changes in specific genes, miRNAs, tyrosine kinase deregulations, and more. Huge amounts of data have been generated that need proper analysis [1]. Several molecular markers with promising prognostic value in PTC patients have been found, including BRAF, NRAS mutation and TERT promoter mutations [2-4]. The V600E mutation represents the vast majority of $B R A F$ mutations, whereas mutations in NRAS codon 61 (Q61R or $\mathrm{Q} 61 \mathrm{~K}$ ) are the most prevalent among RAS gene family mutations [5]. Their prognostic value has been assessed in a number of studies. According to many authors BRAF mutations seem to be associated with markers of clinical aggressiveness (larger tumors, older age, extrathyroidal extension, and LNM), poor clinical outcome and bad response to RAI therapy $[6,7]$. On the other hand, BRAF mutations have not been associated with distant metastases [3, 8].

The prognostic role of RAS mutations is not yet established. Some studies show that their presence is usually associated with distant metastases [9].

Since its first report in $2013[10,11]$ the TERT promoter mutations have been studied intensively in a large number of studies. It became clear that there are two TERT promoter mutations in human cancers - C228T and C250T. As initially demonstrated [11], the TERT C228T and C250T mutations are mutually exclusive and the C250T mutation is far more prevalent in thyroid cancer. Most of the studies have shown that TERT promoter mutations is associated with poor clinicopathological outcomes [10, 12, 13]. Several studies have proven that coexistence of BRAF V600E and TERT promoter mutations constitutes a unique genetic background that drives particularly aggressive pathogenesis and poor clinical outcomes of PTC [14, 15]. One theory to explain this synergistic effect of coexisting BRAF V600E and TERT promoter mutations is that, the BRAF V600Eacctivated MAP kinase pathway upregulates the ETS transcriptional factors. The latter can then bind to the consensus binding site in the TERT promoter created by $\mathrm{C} 228 \mathrm{~T}$ or $\mathrm{C} 250 \mathrm{~T}$ mutation to robustly upregulate the expression of TERT. Then this highly expressed TERT would play a profound tumor-promoting role in thyroid cancer [14]. In recent years, a new trend has emerged in TERT promotor research. TERTlocus single nucleotide polymorphisms (SNPs), lo- cated on chromosome $5 \mathrm{p} 15.33$, have been associated with the risk of several types of human cancers $[16,17]$. The polymorphism rs2853669 in the TERT promoter was identified on chr5:g.1295234A > G (hg38); NM_198253.2(TERT): C.-245T > C) and was shown to be associated with cancer risk in a different populations [18]. A TERT promoter mutation creates a putative binding site for Ets/TCF transcription factors, increasing telomerase activity. In contrast, the TERT rs2853669 variant disrupts Ets/TCF binding [19]. Also, the TERT rs2853669 T > C polymorphism (SNP), located upstream of the TERT promoter region, has been shown to affect telomerase activity and telomere length. The association between TERT rs2853669 and cancer risk has since been studied in multiple ethnicities and populations with inconclusive results, but not in Bulgarian patients. For these reasons, the goal of our study was to analyze $B R A F$, NRAS, single nucleotide polymorphism rs2853669 and TERT (C228T, C250T) in Bulgarian patients with PTC and examine their relationship with clinicopathologic factors.

\section{MATERIAL AND METHODS}

\section{Ethical approval}

All procedures performed in the study were in accordance with the ethical standards of the Bulgarian national research committee and with the 1964 Helsinki declaration and its later amendments or comparable ethical standards. The study was approved by the Ethics Committee of USBALE "Acad. Iv. Penchev" Hospital and written informed consent was signed by all participating patients.

\section{Study design}

The study included 50 patients treated for papillary thyroid carcinoma (PTC) in the Department of Endocrine Surgery, USBALE "Acad. Iv. Penchev" Hospital for the period January 2017 - December 2018 (Table 1). Only the papillary thyroid cancer patients with tumor diameter above or equal to $1 \mathrm{~cm}$ were included in this study. They were analyzed based on the following features: 1 ) sex; 2 ) hereditary thyroid pathology (malignant or benign); 3) histology of the cancer; 4) subtype of PTC; 5) time of diagnosis (age of the patient); 6) the spread of the tumor in the gland (multifocality); 7) The size of the tumor; 8) tumor spread to the lymph nodes (lymph metastasis); 9) the presence of distant metastases; 10) stage of the disease. DNA was isolated from either fresh frozen (20 patients) or FFPE (30 patients) tumors. The fresh frozen samples were taken during the operation of the patients. Half of the tumor was frozen in $-86^{\circ} \mathrm{C}$ fridge for preservation and the 
other half was send for histopathological diagnosis. As for the FFPE samples each was reviewed and the diagnosis was confirmed before DNA isolation. All samples were analyzed for hot spot mutations in BRAF (V600E), NRAS (Q61K), single nucleotide polymorphism (SNP) rs2853669 and TERT (C228T and C250T) genes. Mutation analysis was carried out by Sanger sequencing using automated Genetic Analyzer ABI 3130xI. The primers used for sequencing are shown in Table 2. The results were interpreted using Benchling and SeqScape software. Preoperatively all patients in our series underwent ultrasonographic examination and qualitative evaluation of their nodules was performed by a certified endocrinologist. Furthermore, size, location, and multicentricity of their carcinomas and lymph node metastasis were also evaluated on ultrasonography. From the 50 patients with papillary thyroid cancer 47 underwent preoperative ultrasound-guided fine needle aspiration (FNA) with positive result for PTC. The final diagnosis of all patients was established by histopathology and the thyroid cancer was staged in accordance to the eighth edition AJCC TNM classification for differentiated thyroid carcinoma. From 50 patients with PTC, 45 were in stage I or II and did not require any postoperative treatment. The other 5 patients underwent radioiodine ablation therapy using $30 \mathrm{mCi}$ or more.

\section{Statistical analysis}

The data analysis and interpretation was performed using SPSS 20.0 software package (SPSS Inc., Chicago, IL). The difference between groups was analyzed with chi square test and $p<0.05$ was considered significant.

\section{RESULTS}

The results of the genetic studies for BRAF (V600E), NRAS (Q61K) and TERT (C228T, C250T and SNP rs2853669) mutations are shown in Fig 1 . The distribution of the clinicopathological parameters of the patients and the carrier status for the respective mutation are summarized in Table 3. BRAF (V600E) was detected in 12 out of 50 patients (24\%). The frequency in women with BRAFv600E was $26.3 \%(10 / 38$ women) and $16.66 \%$ in men (2/12 men). When correlating the results obtained for $B R A F(\mathrm{~V} 600 \mathrm{E})$ with the clinical characteristics of the patients, we obtained a statistically significant difference in the size of the tumor (chi2 test $-p=0.038$ ). The analysis showed that in most BRAF (V600E) positive patients the tumor size was between $2-4 \mathrm{~cm}$, in contrast to the negative patients where tumor sizes up to $2 \mathrm{~cm}$ predominated (Fig. 2). Regarding the other clinical characteristics - sex, age, stages, multifocality, lymph metastases, extrathyroid invasion and family history of thyroid dis-

Table 1. Clinical characteristics of the 50 cases with thyroid cancer in the Department of Endocrine Surgery, CCEG, MU-Sofia for the period January 2017 - December 2018

\begin{tabular}{|c|c|c|c|c|c|c|c|}
\hline Gender & $\mathrm{N}$ & \multicolumn{6}{|c|}{ Percentage } \\
\hline Male & 12 & \multicolumn{6}{|c|}{24} \\
\hline Female & 38 & \multicolumn{6}{|c|}{76} \\
\hline Total & 50 & \multicolumn{6}{|c|}{100} \\
\hline Age & $\mathrm{N}$ & \multicolumn{6}{|c|}{ Percentage } \\
\hline Below 45 years & 27 & \multicolumn{6}{|c|}{54} \\
\hline Above 45 years & 23 & \multicolumn{6}{|c|}{46} \\
\hline Total & 50 & \multicolumn{6}{|c|}{100} \\
\hline \multirow{2}{*}{ Histological subtype of Papillary thyroid cancer } & \multirow{2}{*}{$\mathrm{N}$} & \multirow{2}{*}{$\%$} & \multirow{2}{*}{ Multifocal tumors $\mathrm{n}$} & \multicolumn{4}{|c|}{ Stage } \\
\hline & & & & $\mathrm{I}$ & II & III & IV \\
\hline Classical variant & 44 & 88 & 4 & 38 & 3 & 3 & 0 \\
\hline Follicular variant & 6 & 12 & 3 & 4 & 0 & 1 & 1 \\
\hline Total & 50 & 100 & 7 & 42 & 3 & 4 & 1 \\
\hline
\end{tabular}

Table 2. Primers used for Sanger sequencing of the selected mutations RAF (V600E), NRAS (Q61K) and TERT (C228T, C250T and SNP rs2853669)

\begin{tabular}{|c|c|}
\hline Genes & Primers \\
\hline NRAS_ex2F & 5'-GGCAGAAATGGGCTTGAATA-3' \\
\hline NRAS_ex2F & 5'AACCTAAACCAACTCTTCCCATA-3' \\
\hline promTERT-F & 5' GGCCGATTCGACCTCTCT 3' \\
\hline promTERT-R & 5'AGCACCTCGCGGTAGTGG 3' \\
\hline BRAF_ex15_F & 5'AGTAACTCAGCAGCATCTCAG 3' \\
\hline BRAF_ex15_R & 5'AGTAACTCAGCAGCATCTCAG 3' \\
\hline
\end{tabular}


ease (benignant or malignant) no statistically significant correlation was found.

Mutations in the TERT promoter were quite rare in the study group. C228T was detected in one patient $(2 \%)$, while C250T was not detected in the test samples. The BRAF (V600E) mutation was also detected in the only patient with C228T. Unfortunately, this fact precluded further statistical processing. No positive samples were found for NRAS (Q61K) either.

Due to poor sample quality of the FFPE samples, SNP rs2853669 genotype was not obtained for 16 of 50 patients. Rare allele $\mathrm{G}$ of rs2853669 was found in 18 out of 34 patients (52.49\%). A homozygous genotype G/G of rs2853669 was found in 5 patients with papillary thyroid carcinoma and heterozygous genotype in the remaining 13 patients. The minor allele $\mathrm{G}$ frequency in the studied sample was $33,82 \%$. The frequency of the polymorphism was $39.4 \%$ (15/36) among women and $25 \%$ in men (3/8 men). Eleven $(60.5 \%)$ of the 19 patients under 45 years of age were carriers of rs2853669 allele $G$ compared to only five of the 15 patients over 45 years of age. When correlating the rs2853669 genotype with the clinical characteristics of the patient, we did find a statistically significant association with the size of the tumor (chi2 test $-p$
$=0.042$ ). The analysis showed that in patients, either homozygous or heterozygous carriers of allele $G$ the tumor size was less than $2 \mathrm{~cm}$ in diameter, in contrast to the patients with wild type alleles, where tumor size was above $2 \mathrm{~cm}$ (Fig. 3). Regarding the other clinical signs - sex, age, stages, multifocality, lymph metastases, extrathyroid invasion and family history of thyroid disease (benignant or malignant), we were unable to establish statistically significant correlation. SNP rs2853669 co-occurred with BRAF (V600E) mutation in three patients. When we compared the clinical characteristics of these three patients with the other groups, we did not find any significant difference.

\section{DISCUSSION}

The main prognostic factors currently used for the staging and treatment of patients with PTC depend on the final histopathological assessment. This means that the preoperative diagnosis and optimal treatment are still a great challenge. The mutations in BRAF (V600E), NRAS (Q61K) and TERT (C228T, C250T) have been proposed as a potential preoperative marker for risk assessment of patients with papillary thyroid carcinoma (PTC). Many au-

Table 3. Distribution of the frequencies of BRAF (V600E), NRAS (Q61K) and TERT (C228T, C250T and SNP rs2853669) and the clinical features of the patients

\begin{tabular}{|l|c|c|c|c|c|}
\hline & BRAF (V600E) & NRAS(Q61) & TERT (C228T) & TERT (C250T) & TERT (rs2853669) \\
\hline Frequency & $24 \%(12 / 50)$ & $0 \%$ & $2 \%(1 / 50)$ & $0 \%$ & $36 \%(18 / 34)$ \\
\hline Gender & & & & & \\
Male & $16.66 \%(2 / 12)$ & $0 \%$ & $8.3 \%$ & $0 \%$ & $37.5 \%$ \\
& $26.3 \%(10 / 38)$ & & $(1 / 12)$ & & $(3 / 8)$ \\
Female & & $0 \%$ & $0 \%$ & $0 \%$ & $41.6 \%(15 / 36)$ \\
\hline Age & & & & & \\
$<45$ years & $50 \%(6 / 12)$ & $0 \%$ & $0 \%$ & $0 \%$ & $38.9 \%(7 / 18)$ \\
$>\mathbf{4 5}$ years & $50 \%(6 / 12)$ & & $100 \%$ & & $61.1 \%(11 / 18)$ \\
\hline Size & $33.3 \%(4 / 12)$ & $0 \%$ & & & $14 / 18$ \\
$<2 \mathrm{~cm}$ & $66.7 \%(8 / 12)$ & & $100 \%$ & & $4 / 18$ \\
$2-4 \mathrm{~cm}$ & $0 \%$ & & & & $0 \%$ \\
$>\mathbf{4} \mathrm{cm}$ & $8.33 \%(1 / 12)$ & 0 & 0 & 0 & $16 \%(3 / 18)$ \\
\hline Multifocality & & & & & $77.7 \%(16)$ \\
\hline Stage & $66.6 \%(8 / 12)$ & $0 \%$ & $0 \%$ & $0 \%$ & $5.55 \%(1)$ \\
I & $16.6 \%(2 / 12)$ & & $0 \%$ & & $5.55 \%(1)$ \\
II & $16.6 \%(2 / 12)$ & & $100 \%$ & & 0 \\
\hline III & 0 & & $0 \%$ & & $5.55 \%(1 / 28)$ \\
IV & $8.3 \%(1 / 12)$ & $0 \%$ & $0 \%$ & $0 \%$ & $27.7 \%(5 / 18)$ \\
\hline Extrathyroidal extension & $25 \%(3 / 12)$ & $0 \%$ & $100 \%$ & $0 \%$ & \\
\hline Lymph node metastasis & & & &
\end{tabular}




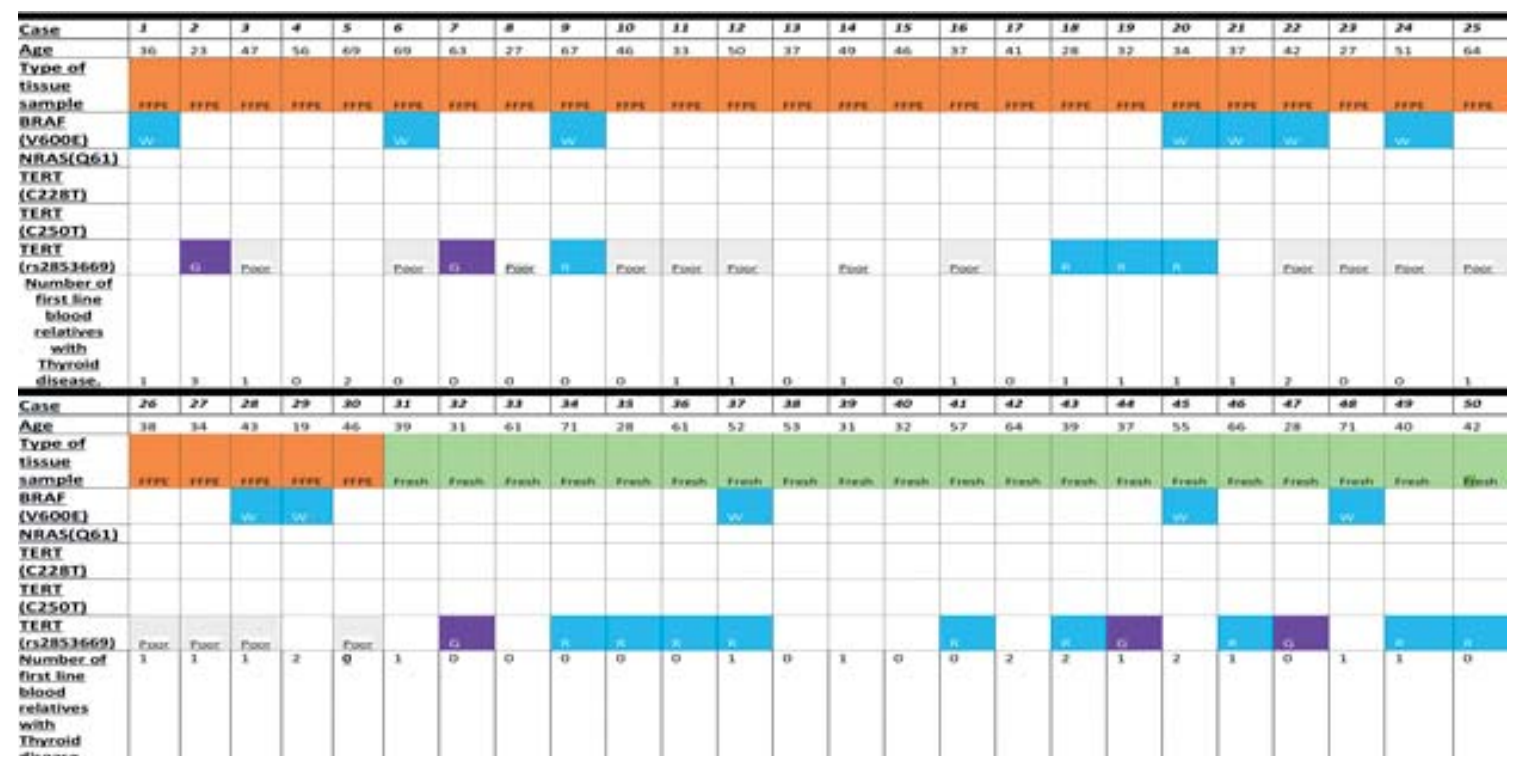

Fig 1. Results of genetic analyses of all patients

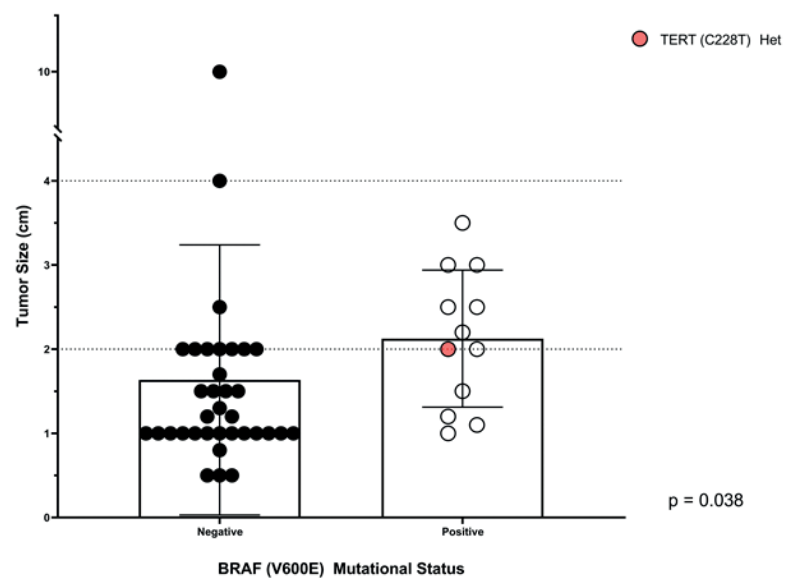

Legend: black circles WT (T/T); white circles heterozygotes (T/C); red circle heterozygote for BRAF(V600E) (T/A) and heterozygote for TERT (C228T)

Fig. 2. Correlation between the tumor size and $B R A F(\mathrm{~V} 600 \mathrm{E})$

thors believe that they can guide the endocrinologists in the selection of best therapeutic strategy and choose between radical surgery or more gentle approach [20-22]. A number of studies have shown that the presence of the BRAF V600E mutation is associated with a poor prognosis in PTC, although in a number of studies there are noticeable discrepancies [23]. A recent study found no association between the BRAF V600E mutation and either aggressive clinicopathological features or persistent or recurrent disease [24].

All the authors agree that the frequency of $B R A F$ V600E has grown in recent years. This leads to the conclusion that this mutation is gradually becoming a concomitant phenomenon for patients with PTC. Proof of this is the comparison: for the period 1990-

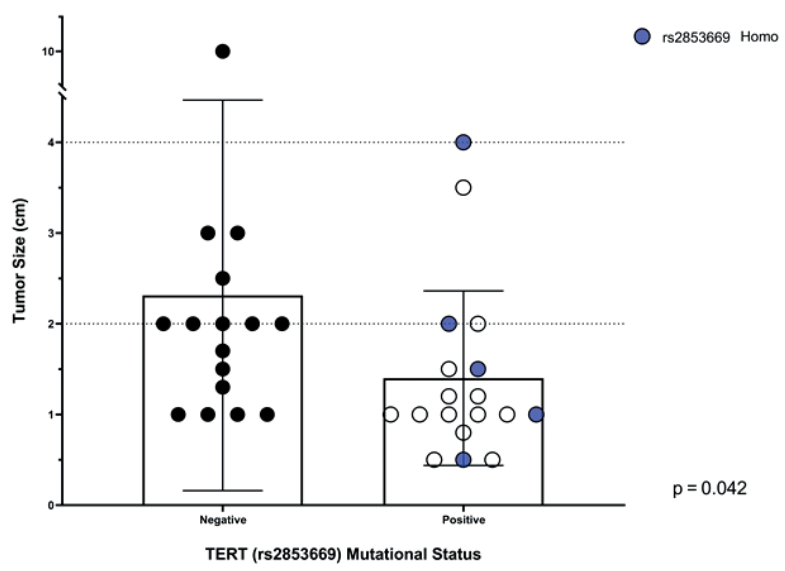

Legend: black circles WT (T/T); white circles heterozygotes (T/C); blue circles homozygotes $(\mathrm{C} / \mathrm{C})$

Fig. 3. Correlation between tumor size and SNP rs2853669

2012 Xing $[22,25]$ and al. reported a BRAF V600E percentage of $38.3 \%$ in patients, while in 2007 Electron et al. [26] reported that the prevalence of $B R A F$ V600E was $46.4 \%$, and in 2012 Kurtulmus et al. [27] showed a BRAF V600E mutation of $39.45 \%$ in PTCs. New data from 2016 [28] advanced tumor stage and lymph node ( $L N$ show that mutations in BRAF V600E are present in $83.7 \%$ of patients with PTC (2789 out of 3332). The reasons for this trend may be the wider access to molecular testing and increased number of tested patients. However, another explanation could be the accumulation of mutations with the advanced age of patients, resulting from the increased life expectancy and the aging of the world's population. In our study, there was no high percentage of BRAF V600E carriers - only $24 \%$. An explanation for this 
fact may be the prevalence of patients under 45 years (27 patients under 45 years and 23 patients over 45 years) in the study group. The role of BRAF V600E mutations in aggressive disease has been extensively studied before [3, 25, 29]. Our results showed correlation only with the size of the primary tumor. We did not find a statistically significant difference for the rest far more important risk features such as multifocality, extrathyroid invasion, lymph metastases [30]. Therefore, the presence of BRAF V600E may not play such an aggressive role in the poor outcomes of patients with PTC. A recent publication questioned the relationship between the BRAF V600E mutation and the prognosis in PTC. Henke et al. found that the mutation did not predict a long-term outcome in PTC [31]. Another explanation is the comparatively small group under investigation and the lack of statistical power to detect significant associations. Nevertheless, the presence of BRAF V600E should be taken into account when individualizing the operative strategy in patients [32].

Single nucleotide polymorphisms can be two types: coding and non-coding SNPs. Noncoders are regulatory, untranslated, intron, silent, and genomic SNPs. Regulatory SNPs (rSNP) are found in non-coding sequences such as promoters, enhancers and the 3 'terms of genes [33]. Some regulatory SNP are likely to affect the expression of neighboring genes [34, 35]. In this way, they affect the tumorigenesis or growth of some cancers. Rs2853669 is such a regulatory SNP. It exists within the promoter of TERT gene. Previous report links this polymorphism with significantly high risk of death and recurrence in patients with liver cancer [36]. Recent meta-analysis demonstrated that rs2853669 did not increase or decrease the overall cancer risk and is not associated with overall cancer prognosis in various type of tumors [18]. However, a modifying effect of rs2853669 on TERTp mutations was revealed among cancer patients with TERTp mutations. Only those carrying the TT genotype had a poor survival and this was supported also by subgroup analyses by cancer type [18]. It is known that the rs2853669 polymorphism is located within a preexisting Ets2 transcription factor binding site in the promoter region of the TERT gene [37]. Therefore, the $\mathrm{C}$ allele variant may impair the Ets2 binding site and then prevent $\mathrm{c}-\mathrm{Myc}$ from binding to the TERT E-box. This factor decreases TERT expression and lowers telomerase activity, thus blunting the harmful effect of TERTp mutations. Therefore, the effect of activating TERTp mutation should be evaluated also on the background of the genotype of rs2853669 polymorphism.
There are currently a few studies of this polymorphism in thyroid cancer. One such study is the study by Muzza et al. [15], which analyzes a sample of the Italian population with thyroid cancer. Their data show an overall frequency of rs2853669 in thyroid carcinomas of $44.4 \%$. They did not show statistically significant differences in clinical signs in the studied patients. A recent study from Japan also examined this polymorphism [38] was that rs2853669 was not associated with PTC tumorigenesis. The high frequency of the rare allele $\mathrm{C}$ of rs2853669 in patients with larger PTC tumors $(81.8 \%)$ suggested that it is related with the growth of PTC.

Our results show the opposite. We have more patients, with the mutant minor allele for rs2853669 with tumor size below $2 \mathrm{~cm}$ in diameter. We should note the differences between our study and the Japanese one, apart from ethnicity. In our study, the TERT mutations were quite rare (only one patient with C228T - 2\%), while in the Japanese PTC patients the frequency was $17.2 \%$ for C228T and $5.2 \%$ for C250T, respectively [38]. They also described two patients with PTC with both the C228T and C250T mutations [38]. Our findings of low frequency of TERT promotor mutations in PTC patients are in agreement with the literature [39-41] and further point to the need of evaluating their prognostic effect together with the genotype of the promotor polymorphism rs2853669, as the variant minor allele $\mathrm{C}$ may blunt the activating effect of the TERTp mutation. The significance of these findings awaits further investigations in larger samples. Most likely, the effect of rs2853669 depends also on the frequency of TERTp mutations in different cancers, and will demonstrate a tissue-specific effect on cancer risk.

Some studies show that PTCs with concurrent BRAF and TERT promoter mutations are associated with increased tumour aggressiveness in comparison with PTCs harbouring BRAF or TERT promoter mutation alone [42]. In our study there was only one patient with such a combination. He had an aggressive PTC cancer with lymph node metastasis in the central compartment and because of the advanced stage he underwent postoperative radioiodine ablation therapy.

\section{CONCLUSION}

In the present study, we demonstrated the association of rs2853669 and BRAF V600E mutations with tumor size in Bulgarian patients with PTCs. While the presence of BRAF V600E mutation was associated with large tumors, rs2853669 polymorphism was found in the majority of PCT tumors below $2 \mathrm{~cm}$. In 
agreement with previous studies, the frequency of NRAS and TERT promotor mutations among Bulgarian PTC patients was a rare event. Due to the potentially modifying role of rs2853669 on activating TERTp mutations and the combined effect of BRAF and TERTp mutation on the tumor aggressiveness, the evaluation of their effect should be done concurrently in different tumors.

Disclosure summary: The authors have nothing to disclose.

\section{Acknowledgements}

Funding: The study has received funds by three Research grants - D-148/23.04.2019 of Medical University, Sofia; KP06-H23/9/18.12.2018 NSF, MES and D01-285/17.12.2019 Ministry of Education and Science, Bulgaria

Ethics approval: This study was performed in line with the principles of the Declaration of Helsinki. Approval was granted by the Ethics Committee of USBALE "Acad. IV. Penchev" Hospital (№ 26/2017)

\section{REFERENCES}

1. Dupuy, A., Simon, R.M. Critical Review of Published Microarray Studies for Cancer Outcome and Guidelines on Statistical Analysis and Reporting. JNCI J. Natl. Cancer Inst. 2007,99, 147-157. https://doi.org/10.1093/jnci/djk018

2. Elisei, R., Viola, D., Torregrossa, L et al. The BRAF(V600E) mutation is an independent, poor prognostic factor for the outcome of patients with low-risk intrathyroid papillary thyroid carcinoma: single-institution results from a large cohort study. J Clin Endocrinol Metab. 2012,97, 4390-4398. https://doi. org/10.1210/jc.2012-1775

3. Liu, C., Chen, T., Liu, Z. Associations between BRAF(V600E) and prognostic factors and poor outcomes in papillary thyroid carcinoma: a meta-analysis. World J Surg Oncol. 2016,14, 241. https://doi.org/10.1186/s12957-016-0979-1

4. Wang, N., Xu, D., Sofiadis, A et al. Telomerase-dependent and independent telomere maintenance and its clinical implications in medullary thyroid carcinoma. J Clin Endocrinol Metab. 2014,99, E1571-9. https://doi.org/10.1210/jc.2014-1158

5. Nikiforov, Y.E., Nikiforova, M.N. Molecular genetics and diagnosis of thyroid cancer. Nat Rev Endocrinol. 2011,7, 569-580. https://doi.org/10.1038/nrendo.2011.142

6. Romei, C., Ciampi, R., Faviana, P et al. BRAFV600E mutation, but not RET/PTC rearrangements, is correlated with a lower expression of both thyroperoxidase and sodium iodide symporter genes in papillary thyroid cancer. Endocr Relat Cancer. 2008,15, 511-520. https://doi.org/10.1677/erc-07-0130

7. Riesco-Eizaguirre, G., Gutierrez-Martinez, P., Garcia-Cabezas, M.A et al. The oncogene BRAF V600E is associated with a high risk of recurrence and less differentiated papillary thyroid carcinoma due to the impairment of $\mathrm{Na}+/ /$ - targeting to the membrane. Endocr Relat Cancer. 2006, 13, 257-269. https://doi.org/10.1677/erc.1.01119

8. George, J.R., Henderson, Y.C., Williams, M.D et al. Association of TERT Promoter Mutation, But Not BRAF Mutation,
With Increased Mortality in PTC. J Clin Endocrinol Metab. 2015,100, E1550-9. https://doi.org/10.1210/jc.2015-2690

9. Eloy, C., Santos, J., Soares, P., Sobrinho-Simões, M. The preeminence of growth pattern and invasiveness and the limited influence of BRAF and RAS mutations in the occurrence of papillary thyroid carcinoma lymph node metastases. Virchows Arch. 2011,459, 265.

10. Liu, R., Xing, M. Diagnostic and Prognostic TERT Promoter Mutations in Thyroid Fine Needle Aspiration Biopsy. Endocr. Relat. Cancer. 2014,21, 825.

11. Liu, X., Wu, G., Shan, Y et al. Highly prevalent TERT promoter mutations in bladder cancer and glioblastoma. Cell cycle. 2013,12, 1637-1638.

12. Melo, M., da Rocha, A.G., Vinagre, J et al. TERT promoter mutations are a major indicator of poor outcome in differentiated thyroid carcinomas. J Clin Endocrinol Metab. 2014,99, E754-65. https://doi.org/10.1210/jc.2013-3734

13. Avares, C., Melo, M., Cameselle-Teijeiro, J.M et al. ENDOCRINE TUMOURS: Genetic predictors of thyroid cancer outcome. 2016,174, R117. https://doi.org/10.1530/ eje-15-0605

14. Xing, M., Liu, R., Liu, X et al. BRAF V600E and TERT promoter mutations cooperatively identify the most aggressive papillary thyroid cancer with highest recurrence. J Clin Oncol. 2014, 32, 2718-2726. https://doi.org/10.1200/jco.2014.55.5094

15. Muzza, M., Colombo, C., Rossi, S et al. Telomerase in differentiated thyroid cancer: promoter mutations, expression and Iocalization. Mol. Cell. Endocrinol. 2015,399, 288-295.

16. Kachuri, L., Latifovic, L., Liu, G., Hung, R.J. Systematic review of genetic variation in chromosome 5p15. 33 and telomere length as predictive and prognostic biomarkers for lung cancer. Cancer Epidemiol. Prev. Biomarkers. 2016,25, 15371549.

17. Killedar, A., Stutz, M.D., Sobinoff, A.P et al. A common cancer risk-associated allele in the hTERT locus encodes a dominant negative inhibitor of telomerase. PLoS Genet. 2015,11.

18. Shen, N., Lu, Y., Wang, $X$ et al. Association between rs2853669 in TERT gene and the risk and prognosis of human cancer: a systematic review and meta-analysis. Oncotarget. 2017,8, 50864-50872. https://doi.org/10.18632/oncotarget. 15140

19. Nencha, U., Rahimian, A., Giry, M et al. TERT promoter mutations and rs2853669 polymorphism: prognostic impact and interactions with common alterations in glioblastomas. J. Neurooncol. 2016,126, 441-446.

20. Basolo, F., Torregrossa, L., Giannini, R et al. Correlation between the BRAF V600E mutation and tumor invasiveness in papillary thyroid carcinomas smaller than 20 millimeters: analysis of 1060 cases. J. Clin. Endocrinol. Metab. 2010,95, 4197-4205.

21. Xing, M., Tufano, R.P., Tufaro, A.P et al. Detection of BRAF mutation on fine needle aspiration biopsy specimens: a new diagnostic tool for papillary thyroid cancer. J. Clin. Endocrinol. Metab. 2004,89, 2867-2872.

22. Tufano, R.P., Teixeira, G. V, Bishop, J et al. BRAF mutation in papillary thyroid cancer and its value in tailoring initial treatment: a systematic review and meta-analysis. Med. 2012,91, 274-286. https://doi.org/10.1097/MD.0b013e31826a9c71

23. 23. Kim, J., Giuliano, A.E., Turner, R.R et al. Lymphatic mapping establishes the role of BRAF gene mutation in papillary thyroid carcinoma. Ann. Surg. 2006,244, 799.

24. Yan, C., Huang, M., Li, $X$ et al. Relationship between BRAF V600E and clinical features in papillary thyroid carcinoma. Endocr. Connect. 2019,8, 988-996. https://doi.org/10.1530/ EC-19-0246 
25. Xing, M., Alzahrani, A.S., Carson, K.A et al. Association between BRAF V600E mutation and recurrence of papillary thyroid cancer. J Clin Oncol. 2015,33, 42-50. https://doi. org/10.1200/jco.2014.56.8253

26. Kebebew, E., Weng, J., Bauer, J et al. The prevalence and prognostic value of BRAF mutation in thyroid cancer. Ann. Surg. 2007,246, 461-466. https://doi.org/10.1097/ SLA.0b013e318148563d

27. Kurtulmus, N., Duren, M., Ince, $U$ et al. BRAF(V600E) mutation in Turkish patients with papillary thyroid cancer: strong correlation with indicators of tumor aggressiveness. Endocrine. 2012,42, 404-410. https://doi.org/10.1007/s12020-012-9651-x

28. Kim, S.K., Woo, J.-W., Lee, J.H et al. Chronic lymphocytic thyroiditis and BRAF V600E in papillary thyroid carcinoma. Endocr. Relat. Cancer. 2016,23, 27-34. https://doi.org/10.1530/ ERC-15-0408

29. Kim, T.H., Park, Y.J., Lim, J.A et al. The association of the BRAF(V600E) mutation with prognostic factors and poor clinical outcome in papillary thyroid cancer: a meta-analysis. Cancer. 2012,118, 1764-1773. https://doi.org/10.1002/cncr.26500

30. Haugen, B.R. 2015 American Thyroid Association Management Guidelines for Adult Patients with Thyroid Nodules and Differentiated Thyroid Cancer: What is new and what has changed? Cancer. 2017,123, 372-381. https://doi. org/10.1002/cncr.30360

31. Henke, L.E., Pfeifer, J.D., Ma, C et al. BRAF mutation is not predictive of long-term outcome in papillary thyroid carcinoma. Cancer Med. 2015,4, 791-799. https://doi.org/10.1002/cam4.417

32. Wang, T.S., Sosa, J.A. Thyroid surgery for differentiated thyroid cancer - recent advances and future directions. Nat. Rev. Endocrinol. 2018,14, 670-683. https://doi.org/10.1038/ s41574-018-0080-7

33. Li, G., Pan, T., Guo, D., Li, L.-C. Regulatory Variants and Disease: The E-Cadherin -160C/A SNP as an Example. Mol. Biol. Int. 2014,2014, 967565. https://doi.org/10.1155/2014/967565

34. Epstein, D.J. Cis-regulatory mutations in human disease. Brief. Funct. Genomic. Proteomic. 2009,8, 310-316. https:// doi.org/10.1093/bfgp/elp021
35. Heidenreich, B., Kumar, R. Altered TERT promoter and other genomic regulatory elements: occurrence and impact. 2017,141, 867-876. https://doi.org/10.1002/ijc.30735

36. Ko, E., Seo, H.-W., Jung, E.S et al. The TERT promoter SNP rs2853669 decreases E2F1 transcription factor binding and increases mortality and recurrence risks in liver cancer. Oncotarget. 2016,7, 684-699. https://doi.org/10.18632/oncotarget.6331

37. Rachakonda, P.S., Hosen, I., de Verdier, P.J et al. TERT promoter mutations in bladder cancer affect patient survival and disease recurrence through modification by a common polymorphism. Proc. Natl. Acad. Sci. U. S. A. 2013,110, 1742617431. https://doi.org/10.1073/pnas.1310522110

38. Hirokawa, T., Arimasu, Y., Chiba, T et al. Clinicopathological significance of the single nucleotide polymorphism, rs2853669 within the TERT promoter in papillary thyroid carcinoma. Pathol. Int. 2020,70, 217-223. https://doi.org/10.1111/ pin. 12900

39. Argyropoulou, M., Veskoukis, A.S., Karanatsiou, P.-M et al. Low Prevalence of TERT Promoter, BRAF and RAS Mutations in Papillary Thyroid Cancer in the Greek Population. Pathol. Oncol. Res. 2020,26, 347-354. https://doi.org/10.1007/ s12253-018-0497-2

40. Qasem, E., Murugan, A.K., Al-Hindi, $\mathrm{H}$ et al. TERT promoter mutations in thyroid cancer: a report from a Middle Eastern population. Endocr. Relat. Cancer. 2015,22, 901-908. https:// doi.org/10.1530/ERC-15-0396

41. Huang, M., Yan, C., Xiao, J et al. Relevance and clinicopathologic relationship of BRAF V600E, TERT and NRAS mutations for papillary thyroid carcinoma patients in Northwest China. Diagn. Pathol. 2019,14, 74. https://doi.org/10.1186/ s13000-019-0849-6

42. Vuong, H.G., Altibi, A.M.A., Duong, U.N.P., Hassell, L. Prognostic implication of BRAF and TERT promoter mutation combination in papillary thyroid carcinoma-A meta-analysis. Clin. Endocrinol. (Oxf). 2017, 87, 411-417. https://doi.org/10.1111/ cen. 13413 